\title{
Upregulated microRNA-210-3p Improves \\ Sevoflurane-induced Protective Effect on Ventricular Remodeling in Rats With Myocardial Infarction by Inhibiting ADCY9
}

\section{Yahui Wu ( $\nabla$ Wuyahui233@163.com )}

Department of Anesthesiology, Henan Provincial People's Hospital, Department of Anesthesiology of Central China Fuwai Hospital of Zhengzhou University

\section{Taofu Wang}

Department of Anesthesiology, Henan Provincial Peoples's Hospital, Department of Anesthesiology of Central China Fuwai Hospital of Zhengzhou University

\section{Liang Qiao}

Department of Otorhinnolaryngology, Henan Provincial Hospital of Zhengzhou University

Hongqi Lin

Department of Anesthesiology, Henan Provincial People's Hospital, Department of Anesthesiology of Central China Fuwai Hospital

\section{Research Article}

Keywords: Myocardial infarction, Ventricular remodeling, Sevoflurane, MicroRNA-210-3p, CUGBP, Adenylyl cyclase type 9

Posted Date: July 26th, 2021

DOl: https://doi.org/10.21203/rs.3.rs-680443/v1

License: (c) (i) This work is licensed under a Creative Commons Attribution 4.0 International License. Read Full License

Version of Record: A version of this preprint was published at Functional \& Integrative Genomics on January 6th, 2022. See the published version at https://doi.org/10.1007/s10142-021-00816-6. 


\section{Abstract}

Objective

Myocardial infarction (MI) is a significant cause of death and disability, and sevoflurane (sevo) can protect myocardium in clinic. We aim to assess the effects of miR-210-3p on MI rats undergoing sevo treatment with the involvement of adenylyl cyclase type 9 (ADCY9).

Methods

Rat MI models were constructed by ligation of the left anterior descending and the modeled mice were respectively treated with sevo, miR-210-3p agomir/antagomir or overexpressed ADCY9. Then, miR-210-3p and ADCY9 expression, cardiac function, myocardial injury and fibrosis, and cardiomyocyte apoptosis in rats were evaluated. Target relation between miR-210-3p and ADCY9 was detected.

Results

MiR-210-3p was downregulated while ADCY9 was upregulated in MI rats. Sevo was able to promote cardiac function and attenuate myocardial injury and fibrosis, as well as cardiomyocyte apoptosis in MI rats. These effects of sevo were strengthened by miR-210-3p elevation while abolished by miR-210-3p inhibition. The role of elevated miR-210-3p in MI rats was reversed by overexpression of ADCY9.

Conclusion

Upregulated miR-210-3p improves sevo-induced protective effect on ventricular remodeling in rats with MI through inhibiting ADCY9.

\section{Introduction}

Myocardial infarction (MI) is mostly caused by rupture of atherosclerotic plaques leading to thrombus formation in lumen of a coronary vessel, which in turn blocks the blood flow to distal myocardium [1]. MI is a life-threatening disease with a high mortality rate that appears not only in developed countries but also in industrialized developing countries [2]. In pathology, MI is defined as cardiomyocyte apoptosis resulting from the prolonged ischemia [3]. Stents were used to treat $\mathrm{Ml}$, which open the occluded coronary artery. Nevertheless, when stents are insufficient, coronary bypass is performed by cardiac pulmonary bypass surgery to maintain regular nourishment of the heart [4]. Although 30-day mortality rate of MI has reduced in the last two decades, it still stays at 7.8\% [5]. Therefore, it is urgent to explore novel biomarkers for the treatment of MI.

Sevoflurane (sevo, 1,1,1,3,3,3-hexafluoro-2-[fluoromethoxy] propane), a highly fluorinated methyl isopropyl ether, was utilized as an inhalational anesthetic for induction and maintenance of general anesthesia [5]. Although basic and clinical researches have suggested that the anesthetic postconditioning of sevo is an effective strategy to ameliorate myocardial injury $[6,7]$, the detailed mechanism has not been completely 
explored. MicroRNAs (miRNAs) are small non-coding RNAs that constrain gene expression through binding the 3 '-untranslated region (3'-UTR) and then influence mRNA stability or protein translation [8]. Previous studies have shown that sevo treatment was able to alter the expression of miRNAs in cardiovascular diseases $[9,10]$. MiR-210-3p is one of the two mature forms for miR-210 which is the most prominent hypoxia regulated miRNAs [11]. MiR-210-3p has been elucidated to show a decreased level in $\mathrm{MI}$ mice [12], while the underlying mechanisms of miR-210-3p on MI as well as the regulatory relation between sevo and miR-210-3p remains rarely explored. Adenylyl cyclase type 9 (ADCY9) is an enzyme producing the ubiquitous second messenger cyclic AMP. In the absence of cholesteryl ester transfer protein activity, ADCY9 inactivation is found to protect against atherosclerosis [13].

This study focused on the effects of sevo treatment and miR-210-3p on ventricular remodeling following MI. We inferred that miR-210-3p may affect the sevo-induced effect on ventricular remodeling in $\mathrm{MI}$ rats with the involvement of ADCY9.

\section{Materials And Methods}

\section{Ethics statement}

Animal experiments were strictly in accordance with the Guide to the Management and Use of Laboratory Animals issued by the National Institutes of Health. The protocol of animal experiments was approved by the Institutional Animal Care and Use Committee of Henan Provincial People's Hospital.

\section{Experimental animals}

Clean and healthy adult male Sprague Dawley rats (aged 8-12 w and weighed 200-300 g) that obtained from Experimental animal center of Xi'an Jiaotong University (Shanxi, China) were fed in standard rat cages capable for ventilation in a quiet environment (natural light, $23-25^{\circ} \mathrm{C}$, relative humidity of $55-$ $60 \%)$. The paddings in cages were changed every day.

\section{Establishment of Ml rat models}

Rats were anesthetized by intraperitoneal injection of $2.5 \%$ pentobarbital sodium $(50 \mathrm{mg} / \mathrm{kg})$, fixed on an operating table in a supine position and intubated with a small animal artificial ventilator (respiratory rate at 50-60 times $/ \mathrm{min}$, tidal volume $=2 \mathrm{~mL} / \mathrm{mg}$ based on body weight). A longitudinal incision was made 1 $\mathrm{cm}$ from the left side of rat sternum to open the thorax and pericardium. With portal coronary vein as the mark, 5/0 suture was inserted $2 \mathrm{~mm}$ below root of the left atrial appendage and reached the arterial cone (3-4 mm from the origin, depth of 1.5-2 mm). A piece of plastic tube (length of $1 \mathrm{~cm}$ ) was pressed down along the double lines to block the blood flow of the left anterior descending branch of the coronary artery (LAD). The gray color of the left ventricular wall and the ST segment elevation of electrocardiogram indicated a complete ligation. Then, the rats were conducted with 30-minute ischemia and 24-h reperfusion [14].

\section{Animal grouping}


The 70 modeled rats were classified into 7 groups $(n=10)$ : the MI, sevo, sevo + antagomir negative control (NC), sevo + miR-210-3p antagomir, sevo + agomir NC, sevo + miR-210-3p agomir, sevo + miR-210$3 p$ agomir + overexpressed (oe)-NC and sevo + miR-210-3p agomir + oe-ADCY9 groups. Rats in the MI group were performed with MI model establishment and those in the sevo group were conducted with inhalation of $3.4 \%$ sevo for 5 min before the reperfusion. Rats in other groups were respectively intramyocardially injected with lentivirus $\left(5 \times 10^{7} \mathrm{TU} / \mathrm{mL}\right)$ carrying NC of miR-210-3p antagomir, lentivirus $\left(5 \times 10^{7} \mathrm{TU} / \mathrm{mL}\right)$ carrying miR-210-3p antagomir, lentivirus $\left(5 \times 10^{7} \mathrm{TU} / \mathrm{mL}\right)$ carrying NC of miR-210-3p agomir, lentivirus $\left(5 \times 10^{7} \mathrm{TU} / \mathrm{mL}\right)$ carrying miR-210-3p agomir, and lentivirus $\left(5 \times 10^{7} \mathrm{TU} / \mathrm{mL}\right)$ carrying miR-210-3p agomir and $10 \mu \mathrm{g}(100 \mu \mathrm{L})$ overexpressed ADCY9 plasmid 30 min before the surgery. Next, rats were conducted with inhalation of $3.4 \%$ sevo for $5 \mathrm{~min}$ before the reperfusion. Rats in the sham group (LAD was threaded but not ligated) were taken as the controls [10].

\section{Electrocardiogram}

Fourteen days after the surgery, rats were anesthetized by intraperitoneal injection of $2.5 \%$ pentobarbital sodium (40 mg/kg), fixed in a supine position and conducted with the electrocardiograph (Philips SONOS-7500, Philips, Andover, MA, USA, probe frequency at $8.0 \mathrm{MHz}$ ). The long axis and short axis sections of the left ventricle beside the sternum were obtained by gently touching the precordial area with an ultrasonic probe. The left ventricle internal dimension at systole (LVIDs) and diastole (LVIDd) were detected, and the left ventricle ejection fraction (LVEF) and fractional shortening (FS) were calculated $(n=$ 10).

\section{Collection of blood samples and myocardial tissues}

Blood from celiac artery $(n=5)$ was collected before the enthanasia. Hearts $(n=5)$ were harvested and the anterior wall of left ventricle in blood supply area of LAD was isolated. Tissues were used for protein and RNA extraction, hematoxylin-eosin (HE) staining, Masson staining and terminal deoxynucleotidyl transferase-mediated deoxyuridine triphosphate nick end-labeling (TUNEL) staining.

\section{Evaluation of creatine kinase (CK), lactate dehydrogenase (LDH), malondialdehyde (MDA) and superoxide dismutase (SOD) levels}

Serum samples were extracted from rats to assess the activities of CK and LDH using the kits (JianCheng Bioengineering Institute, Jiangsu, China). Tissue homogenate was appropriately diluted and protein concentration was measured with bicinchoninic acid (BCA) kits (Sigma-Aldrich Chemical Company, MO, USA). Then, SOD activity and MDA content were measured based on the kits (JianCheng Bioengineering Institute).

\section{HE staining}

Sections were normally dehydrated with gradient ethanol, permeabilized with xylene, rinsed by deionized water and stained by hematoxylin for 3-5 min, and then differentiated by $1 \%$ hydrochloric alcohol (20 s), 
blued by $1 \%$ ammonium hydroxide ( $30 \mathrm{~s}$ ) and counterstained with $1 \%$ eosin dye solution ( $5 \mathrm{~min}$ ). Next, the sections were normally dehydrated, permeabilized, dried, sealed and observed and photographed through a microscope.

\section{Masson staining}

Sections were normally dehydrated with gradient ethanol, permeabilized with xylene, stained by hematoxylin for $30 \mathrm{~s}$, blued with clear water for $3 \mathrm{~min}$ and soaked in ponceau S acid fuchsin for $3 \mathrm{~min}$, and then differentiated with $1 \%$ phosphomolybdate acid (twice, $3 \mathrm{~min} /$ time), counterstained with $1 \%$ aniline blue for $5 \mathrm{~min}$ and differentiated by $1 \%$ glacial acetic acid for $10 \mathrm{~s}$. Then, the sections were permeabilized, sealed and conducted with microscopy observation. An image analysis system was used for semi-quantitative analysis of myocardial collagens and the collagen volume fraction (CVF) was calculated. Five random fields of view were selected for the calculation of percentage of collagen tissues in each field, and the mean value was adopted.

\section{TUNEL staining}

Paraffin sections were normally dewaxed and hydrated, and then performed with TUNEL staining with the TUNEL kits (Roche, CA, USA). The sections were developed with diaminobenzidine, counterstained with hematoxylin for 3 min, soaked in ethanol for 1-2 s, and then were dehydrated and permeabilized with xylene and sealed with neutral balsam. Positive standard: nuclei of apoptotic cardiomyocytes were brownish-yellow under a light microscope.

\section{Reverse transcription quantitative polymerase chain reaction (RT-qPCR)}

Trizol kits were used to extract total RNA in myocardial tissues and the RNA was reversely transcribed into cDNA based on directions of RNA reverse transcription kits (TaKaRa Biotechnology Co., Ltd., Liaoning, China). The cDNA was conducted with PCR amplification and the products were confirmed by agarose gel electrophoresis. Data were analyzed using $2-\Delta \Delta C t$ method and the PCR primers (Table 1) were synthesized by TaKaRa. U6 was used as the internal reference for miR-210-3p and glyceraldehyde phosphate dehydrogenase (GAPDH) was taken as the internal reference for ADCY9, collagen I and collagen III. 
Table 1

Primer sequence

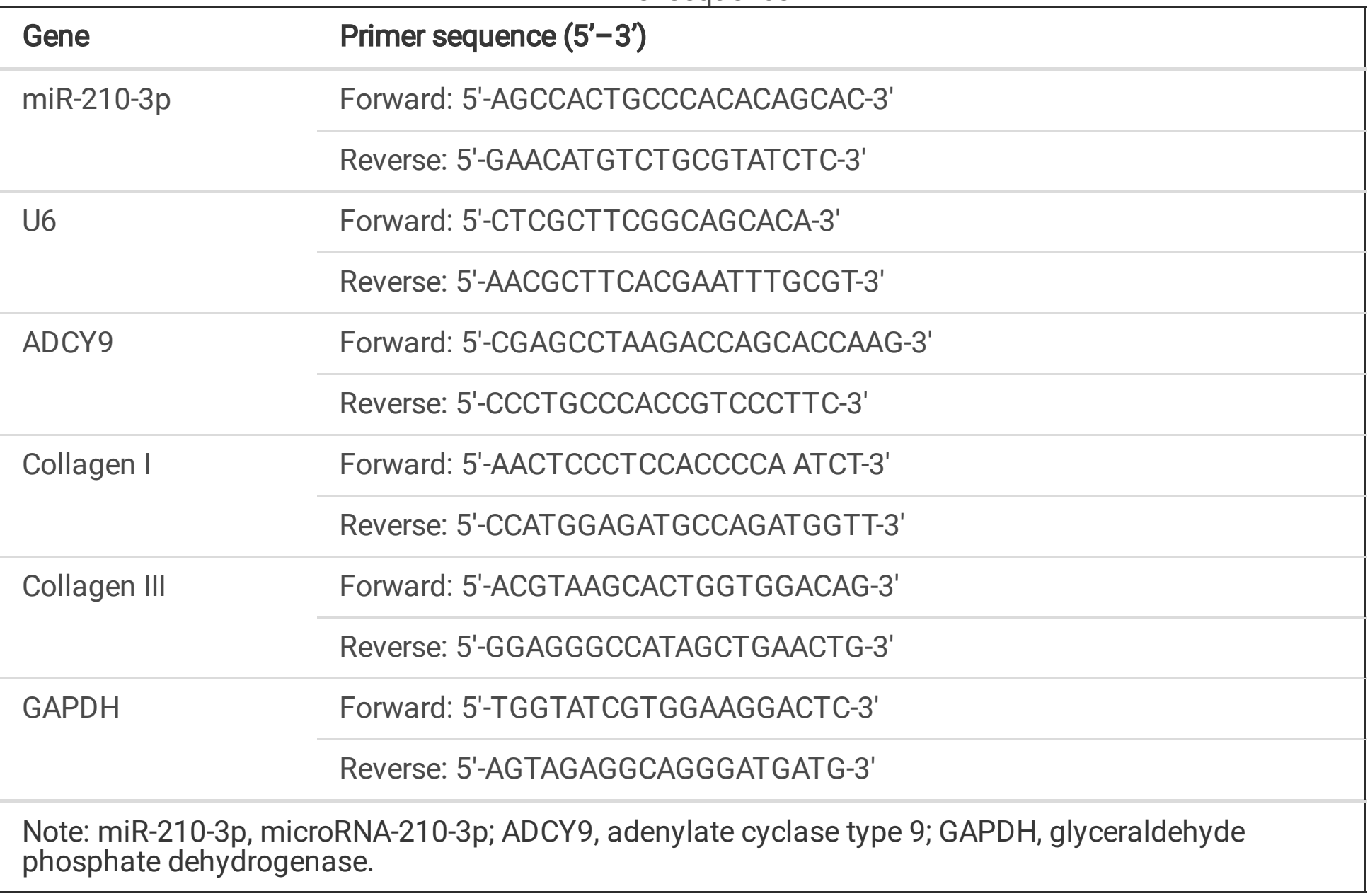

\section{Western blot analysis}

Total protein in myocardial tissues was extracted. The proteins were treated with $10 \%$ sodium dodecyl sulfate-polyacrylamide gel electrophoresis for $2 \mathrm{~h}$ and transferred onto membranes, which were blocked with $5 \%$ skim milk powder for $2 \mathrm{~h}$. Afterwards, the membranes were incubated with primary antibodies ADCY9, Collagen I, Collagen III and GAPDH (all 1: 1000 and from Santa Cruz Biotechnology, Inc, CA, USA), and then incubated with relative secondary antibody. Enhanced chemiluminescent method was used for development and the optical density values of bands were measured with GAPDH as the loading control. The ratio of OD value of target band to that of GAPDH was the relative expression of target proteins.

\section{Dual luciferase reporter gene assay}

Target relation between miR-210-3p and ADCY9, and the biding sites of miR-210-3p and ADCY9 3'UTR were predicted by an online prediction software (https://cm.jefferson.edu/). ADCY9 3'UTR was amplified and the wild type (WT) and mutant type (MUT) recombinant plasmids were respectively extracted based on protocols of plasmid extraction kits (Invitrogen Inc., CA, USA). The plasmids were digested by restriction enzymes Xba I and Xho I. The products were purified, recycled and connected with luciferase report vector pmir-GLO by T4DNA ligase, and then were transferred to DH5a competent Escherichia coli. Plasmids were extracted, confirmed by $\mathrm{Xba} \bigotimes$ and $\mathrm{Xho} \otimes$ double digestion and sequenced. ADCY9 WT 
(ADCY9-3'UTR-WT) and MUT (ADCY9-3'UTR-MUT) recombinant dual luciferase report plasmid were constructed. Lipidosome method was used to respectively co-transfect the report gene vectors and miR210-3p mimic and mimic NC into 293T cells (American Type Culture Collection, VA, USA) containing pRLTK vector. The firefly and renilla luciferase activities in cells were determined by a fluorescent detector according to instructions of dual luciferase report gene detection kits (Promega Corporation, WI, USA).

\section{Statistical analysis}

All data analyses were conducted using SPSS 21.0 software (IBM Corp. Armonk, NY, USA). The measurement data conforming to the normal distribution were expressed as mean \pm standard deviation. The unpaired t-test was performed for comparisons between two groups, one-way or two-way analysis of variance (ANOVA) was used for comparisons among multiple groups and Tukey's post hoc test was used for pairwise comparisons after one-way ANOVA. $P$ value $<0.05$ was indicative of statistically significant difference.

\section{Results}

\section{Sevo improves cardiac function of MI rats}

In order to explore the effect of sevo on $\mathrm{Ml}$, we firstly evaluated rat cardiac function using an electrocardiograph, and the results (Fig. 1A-D) indicated that LVIDs and LVIDd were heightened while LVEF and FS were lowered in the MI group versus the sham group (all $P<0.05$ ); rats in the sevo group had suppressed LVIDs and LVIDd and elevated LVEF and FS when compared with the MI group (all $P<0.05$ ). The activities of serum CK and LDH as well as myocardial MDA and SOD were determined and we found that (Fig. 1E-H) versus the sham group, the MI group exhibited increased levels of CK, LDH and MDA and decreased level of SOD (all $P<0.05$ ). In comparison with the Ml group, levels of CK, LDH and MDA were reduced and SOD level was increased in the sevo group (all $P<0.05$ ).

Collagen I and collagen III expression in rats were detected to investigate the role of sevo on myocardial collagen expression in MI rats. The results suggested that (Fig. 1I) collagen I and collagen III were upregulated in the $\mathrm{MI}$ group rather than the sham group (both $P<0.05$ ), while the upregulated collagen I and collagen III were suppressed in rats of the sevo group compared with the MI group (both $P<0.05$ ). Masson staining results (Fig. 1J, K) showed that CVF was significantly increased in Ml group versus the sham group; CVF was significantly decreased in the sevo group compared with the MI group. Results of HE staining (Fig. 1L) implied that in the sham group, myocardial fibers were arranged orderly and there existed clear striation structure without myocardial fiber rupture and inflammatory cell infiltration. In the $\mathrm{Ml}$ group, myocardial fibers were arranged disorderly and there exhibited myocardial fiber rupture, fuzzy striation structure and a large number of inflammatory cells infiltration. In the sevo group, the myocardial injury was improved. TUNEL staining was used to detect the apoptosis of cardiomyocytes and the results (Fig. 1M) showed that versus the sham group, the apoptosis rate of cardiomyocytes in the MI group was significantly increased; contrasted to the MI group, the apoptosis rate of cardiomyocytes in the sevo 
group was significantly decreased (all $P<0.05$ ). These results suggested that sevo can reduce the collagen fibers and cardiomyocyte apoptosis in myocardial tissue of $\mathrm{Ml}$ rats.

\section{MiR-210-3p downregulation eliminates sevo-induced effect on improving $\mathrm{Ml}$ in a rat model}

Here we detected miR-210-3p expression in MI rat myocardial tissues to explore its regulatory role in MI. It was found via RT-qPCR that (Fig. 2A) miR-210-3p was downregulated in the MI group versus the sham group; in contrast to the MI group, miR-210-3p was upregulated in the sevo group (all $P<0.05$ ).

In order to detect the effect of sevo on miR-210-3p, we downregulated miR-210-3p in sevo-treated MI rats, and it was verified by RT-qPCR that (Fig. 2B) compared with sevo + antagomir NC group, the expression level of miR-210-3p in myocardial tissue of the sevo + mir-210-3p antagomir group was significantly decreased $(P<0.05)$. Moreover, the cardiac function of rats was evaluated and the results showed that versus the sevo + antagomir NC group, LVIDs and LVIDd in the sevo + miR-210-3p antagomir group were significantly increased, while LVEF and FS were significantly decreased (Fig. 2C-F) (all $P<0.05$ ), indicating that downregulating miR-210-3p reverses the effect of sevo on improving cardiac function in Ml rats.

Serum activities of CK and LDH, as well as myocardial MDA content and SOD activity of the rats were determined. The results suggested that (Fig. 2G-J) in relation to the sevo + antagomir NC group, the levels of CD, LDH and MDA were increased while SOD level was decreased in the sevo + miR-210-3p antagomir group (all $P<0.05$ ). Collagen I and collagen III expression was determined and we found that (Fig. $2 \mathrm{~K}$ ) versus the sevo + antagomir NC group, collagen I and collagen III expression was increased in the sevo + miR-210-3p antagomir group (both $P<0.05$ ). The Masson staining, HE staining and TUNEL staining revealed that (Fig. 2L-O) compared with the sevo + antagomir NC group, the CVF, inflammatory cell infiltration and cardiomyocyte apoptosis were increased in the sevo + miR-210-3p antagomir group (all $P$ $<0.05)$.

The above data implied that miR-210-3p downregulation could eliminate sevo-induced effect on improving $\mathrm{Ml}$ in a rat model.

\section{MiR-210-3p upregulation strengthens sevo-induced effect on improving $\mathrm{Ml}$ in a rat model}

We upregulated miR-210-3p in MI rats to observe its role. Results of RT-qPCR suggested that (Fig. 3A) relative to the sevo + agomir group, miR-210-3p was upregulated in the sevo + miR-210-3p agomir group $(P<0.05)$. We detected the cardiac function of rats and it was found that (Fig. 3B-E) versus the sevo + agomir NC group, LVIDs and LVIDd in the sevo + miR-210-3p antagomir group were reduced, while LVEF and FS were enhanced, implying that upregulating miR-210-3p improves the effect of sevo on promoting cardiac function in $\mathrm{Ml}$ rats (all $P<0.05$ ). 
CK, LDH, MDA and SOD levels of the rats were assessed and we found that (Fig. 3F-I) compared with the sevo + agomir NC group, the levels of CD, LDH and MDA were decreased while SOD level was increased in the sevo + miR-210-3p agomir group (all $P<0.05$ ). Collagen I and collagen III expression was detected and the results showed that (Fig. $3 \mathrm{~J}$ ) versus the sevo + agomir NC group, collagen I and collagen III expression was suppressed in the sevo + miR-210-3p agomir group (both $P<0.05$ ). Results of Masson staining, HE staining and TUNEL staining reflected that (Fig. 3K-N) versus the sevo + agomir NC group, the CVF, inflammatory cell infiltration and cardiomyocyte apoptosis were inhibited in the sevo + miR-210-3p agomir group (all $P<0.05$ ).

These results mirrored that miR-210-3p upregulation could promote sevo-induced effect on improving MI in a rat model.

\section{ADCY9 upregulation reverses the therapeutic effects of sevo and elevated miR-210-3p on Ml rats}

In order to study the effect of ADCY9 on MI in rats treated with sevo + miR-210-3p agomir, we upregulated ADCY9 and verified the alteration using RT-qPCR and Western blot analysis (Fig. 4A, B). It came out that compared with the sevo + miR-210-3p group, ADCY9 expression was enhanced in the sevo + miR-210-3p agomir + oe-ADCY9 group $(P<0.05)$. Next, cardiac function was evaluated and our results indicated that contrasted to the sevo + miR-210-3p agomir group, LVIDs and LVIDd were significantly increased, while LVEF and FS were significantly decreased in the sevo + miR-210-3p agomir + oe-ADCY9 group (Fig. 4C-F) (all $P<0.05$ ), showing that ADCY9 elevation abolishes the effect of sevo and miR-210-3p on improving cardiac function in $\mathrm{MI}$ rats.

Serum activities of CK and LDH, as well as myocardial MDA content and SOD activity of the rats were assessed. We discovered that (Fig. 4G-J) versus the sevo + miR-210-3p agomir group, the levels of CD, LDH and MDA were enhanced while SOD level was reduced in the sevo + miR-210-3p agomir + oe-ADCY9 group (all $P<0.05$ ). Results of collagen I and collagen III expression detection reflected that (Fig. $4 \mathrm{~K}$ ) the sevo + miR-210-3p agomir + oe-ADCY9 group had higher collagen I and collagen III expression than the sevo + miR-210-3p agomir group (both $P<0.05$ ). Additionally, it was found from the Masson staining, HE staining and TUNEL staining that (Fig. 4L-O) versus the sevo + miR-210-3p agomir group, the CVF, inflammatory cell infiltration and cardiomyocyte apoptosis were increased in the miR-210-3p agomir +oeADCY9 group (all $P<0.05$ ).

Our results showed that $A D C Y 9$ upregulation reversed the therapeutic effects of sevo and elevated miR210-3p on MI rats.

\section{ADCY9 is a direct target gene of miR-210-3p}

To further study the effect of ADCY9 on miR-210-3p, we detected ADCY9 expression in myocardial tissue of rats using RT-qPCR and Western blot analysis. Results showed that (Fig. 5A-D) compared with the sevo + antagomir NC group, ADCY9 was upregulated in the sevo + miR-210-3p antagomir group $(P<0.05)$. 
In relation to the sevo + agomir NC group, ADCY9 was downregulated in the sevo + miR-210-3p agomir group $(P<0.05)$.

As predicted by a bioinformatic software (https://cm.jefferson.edu/), there existed a binding relationship between miR-210-3p and ADCY9 (Fig. 5E). Outcomes of dual luciferase report gene assay indicated that (Fig. 5F) in ADCY9-3'UTR-WT, the luciferase activity of cells in the miR-210-3p mimic group was lower than that in the mimic NC group $(P<0.01)$; while in ADCY9-3'UTR-MUT, there showed no apparent difference in luciferase activity of cells between the mimic NC group and the miR-210-3p mimic group ( $P$ $>0.05$ ), indicating that ADCY9 is a direct target gene of miR-210-3p and miR-210-3p upregulation could inhibit ADCY9 expression.

\section{Discussion}

Adverse left ventricular remodeling after $\mathrm{Ml}$ is the structural basis for ischemic heart failure and involves complex short- and long-term changes in size, shape, function, and cellular and molecular composition in the left ventricular [15]. We aimed to assess the role of sevo treatment regulating miR-210-3p in ventricular remodeling after $\mathrm{MI}$ with the involvement of $\mathrm{ADCY} 9$, and we found that sevo upregulated miR210-3p to serve as a protective role in ventricular remodeling after MI by inhibiting ADCY9.

In our study, Ml rats were treated with sevo to observe its effects. The results reflected that sevo treatment was able to promote cardiac function and repress myocardial injury and fibrosis, and cardiomyocyte apoptosis in MI rats. Consistently, Zhang et al. have elucidated that sevo contributed to stabilizing the cardiopulmonary function and preventing from myocardial injury in patients undergoing coronary artery bypass [16]. A recent literature has confirmed that sevo improved pulmonary fibrosis in rats with pulmonary arterial hypertension [16]. It has been previously identified that sevo exposure prevented diaphragmatic oxidative stress during mechanical ventilation in a rat model [17], Pasqualin et al. have affirmed that sevo preconditioning reduced infarct size in rats after myocardial I/R [18], and Lu et al. have recently demonstrated that sevo prevented hypoxia/reoxygenation-induced cardiomyocyte apoptosis [19]. Moreover, sevo treatment has been verified to have positive or negative impact on the expression of some particular RNAs [20]. For instance, sevo has been clarified to upregulate miR-203 in colorectal cancer [21], and it has also been revealed that sevo anesthesia positively regulated the expression of miR-34a-3p to increase viability and inhibit apoptosis of lung cells in acute lung injury [22]. However, the mechanisms of sevo treatment affecting expression of miR-210-3p remains unclear.

MiR-210-3p expression in rat myocardial tissues was assessed in our research and it was found that miR210-3p was lowly expressed in myocardial tissues from Ml rats, and the sevo treatment was able to upregulate miR-210-3p in MI rats. In line with this finding, Ma et al. have discovered that miR-210-3p expression was reduced in fat-1 transgenic mice in comparison to WT mice [12], and a former document has unraveled that miR-210 expression was lower in MI rats [23]. Furthermore, sevo preconditioning has been demonstrated to elevate the expression of miR-210 to promote activation of resident cardiac stem cells in a MI rat model [9]. As for the protective role of miR-210-3p, we have found that sevo-induced 
upregulation of miR-210-3p was capable of promoting cardiac function and repressing myocardial injury and fibrosis and cardiomyocyte apoptosis in MI rats. Similarly, Wang et al. have affirmed that miR-210, enriched by mesenchymal stem cells-derived extracellular vesicles, had the ability to promote cardiac function recovery [24], a study has unearthed that miR-210 protected cardiomyocytes against oxygenglucose deprivation/reperfusion [25], and it has been uncovered that the elevation of mesenchymal stem cells-derived exosomal miR-210 reduced fibrosis of cardiac progenitor cells in the infarcted heart [26]. In addition, Arif et al. have mentioned that rodent hearts with miR-210 upregulation performed a decrease in cardiomyocyte apoptosis after myocardial infarction [27], and a recent research has identified that the elevated miR-210 alleviated endothelial oxidative stress [28]. Additionally, the miRNAs were known to bind to the 3'UTR of mRNAs of their target genes [29]. The targeting relationship between miR-210-3p and $A D C Y 9$ was confirmed in our study. We also measured ADCY9 expression in rat myocardial tissues, and it came out that ADCY9 was highly expressed in myocardial tissues from MI rats and overexpression of $A D C Y 9$ reduced the effects of miR-210-3p on MI rats as well. Consistently, Yohann Rautureau et al. have identified that the ADCY9 inactivation is protective on atherosclerosis in the absence of cholesteryl ester transfer protein [13].

In conclusion, we demonstrated that sevo treatment upregulated miR-210-3p to protect from ventricular remodeling after $\mathrm{MI}$ through reducing $\mathrm{ADCY} 9$ expression. This research may deepen the understanding of molecular mechanisms of Ml. However, we did not conduct the in vitro experiment for investigating the detailed mechanisms due to the limited time and fund, thus, more efforts are needed for further investigations.

\section{Declarations}

\section{Funding}

None

\section{Ethics approval and consent to participate}

Animal experiments were strictly in accordance with the Guide to the Management and Use of Laboratory Animals issued by the National Institutes of Health. The protocol of animal experiments was approved by the Institutional Animal Care and Use Committee of Henan Provincial People's Hospital.

\section{Conflict of interest}

The authors declare that they have no conflicts of interest.

\section{Consent for publication}

Not applicable

\section{Availability of data and material}

Not applicable

\section{Authors' contributions}

Yahui Wu contributed to study design; Yahui Wu contributed to manuscript editing; Taofu Wang and Liang Qiao contributed to experimental studies; Hongqi Lin contributed to data analysis. 


\section{Acknowledgement}

We would like to acknowledge the reviewers for their helpful comments on this pap.

\section{References}

1. Curley, D., B. Lavin Plaza, A.M. Shah, and R.M. Botnar, Molecular imaging of cardiac remodelling after myocardial infarction. Basic Res Cardiol, 2018. 113(2): p. 10.

2. Michaud, K., C. Basso, G. d'Amati, C. Giordano, I. Kholova, S.D. Preston, S. Rizzo, S. Sabatasso, M.N. Sheppard, A. Vink, et al., Diagnosis of myocardial infarction at autopsy: AECVP reappraisal in the light of the current clinical classification. Virchows Arch, 2020. 476(2): p. 179-194.

3. Thygesen, K., J.S. Alpert, H.D. White, and E.S.C.A.A.H.A.W.H.F.T.F.f.t.R.o.M.I. Joint, Universal definition of myocardial infarction. J Am Coll Cardiol, 2007. 50(22): p. 2173-95.

4. Aydin, S., K. Ugur, S. Aydin, I. Sahin, and M. Yardim, Biomarkers in acute myocardial infarction: current perspectives. Vasc Health Risk Manag, 2019. 15: p. 1-10.

5. Montrief, T., W.T. Davis, A. Koyfman, and B. Long, Mechanical, inflammatory, and embolic complications of myocardial infarction: An emergency medicine review. Am J Emerg Med, 2019. 37(6): p. 1175-1183.

6. Qiao, S.G., Y. Sun, B. Sun, A. Wang, J. Qiu, L. Hong, J.Z. An, C. Wang, and H.L. Zhang, Sevoflurane postconditioning protects against myocardial ischemia/reperfusion injury by restoring autophagic flux via an NO-dependent mechanism. Acta Pharmacol Sin, 2019. 40(1): p. 35-45.

7. Cao, J., H. Xie, Y. Sun, J. Zhu, M. Ying, S. Qiao, Q. Shao, H. Wu, and C. Wang, Sevoflurane postconditioning reduces rat myocardial ischemia reperfusion injury through an increase in NOS and a decrease in phopshorylated NHE1 levels. Int J Mol Med, 2015. 36(6): p. 1529-37.

8. Yang, M., X. Tang, Z. Wang, X. Wu, D. Tang, and D. Wang, miR-125 inhibits colorectal cancer proliferation and invasion by targeting TAZ. Biosci Rep, 2019. 39(12).

9. Wen, T., L. Wang, X.J. Sun, X. Zhao, G.W. Zhang, and J. Li-Ling, Sevoflurane preconditioning promotes activation of resident CSCs by transplanted BMSCs via miR-210 in a rat model for myocardial infarction. Oncotarget, 2017. 8(70): p. 114637-114647.

10. Qi, Z., S. Li, Y. Su, J. Zhang, Y. Kang, Y. Huang, F. Jin, and Q. Xing, Role of microRNA-145 in protection against myocardial ischemia/reperfusion injury in mice by regulating expression of GZMK with the treatment of sevoflurane. J Cell Physiol, 2019.

11. Pasculli, B., R. Barbano, M. Rendina, A. Fontana, M. Copetti, T. Mazza, V.M. Valori, M. Morritti, E. Maiello, P. Graziano, et al., Hsa-miR-210-3p expression in breast cancer and its putative association with 
worse outcome in patients treated with Docetaxel. Sci Rep, 2019. 9(1): p. 14913.

12. Ma, H., P. Chen, C. Sang, D. Huang, Q. Geng, and L. Wang, Modulation of apoptosis-related microRNAs following myocardial infarction in fat-1 transgenic mice vs wild-type mice. J Cell Mol Med, 2018. 22(11): p. 5698-5707.

13. Rautureau, Y., V. Deschambault, M.E. Higgins, D. Rivas, M. Mecteau, P. Geoffroy, G. Miquel, K. Uy, R. Sanchez, V. Lavoie, et al., ADCY9 (Adenylate Cyclase Type 9) Inactivation Protects From Atherosclerosis Only in the Absence of CETP (Cholesteryl Ester Transfer Protein). Circulation, 2018. 138(16): p. 1677-1692.

14. Ferdinandy, P., R. Schulz, and G.F. Baxter, Interaction of cardiovascular risk factors with myocardial ischemia/reperfusion injury, preconditioning, and postconditioning. Pharmacol Rev, 2007. 59(4): p. 418-58.

15. Prabhu, S.D. and N.G. Frangogiannis, The Biological Basis for Cardiac Repair After Myocardial Infarction: From Inflammation to Fibrosis. Circ Res, 2016. 119(1): p. 91-112.

16. Zhang, J. and S. Wang, Effects of sevoflurane on cardiopulmonary function in patients undergoing coronary artery bypass. J Biol Regul Homeost Agents, 2016. 30(4): p. 1079-1083.

17. Breuer, T., K. Maes, R. Rossaint, G. Marx, H. Scheers, I. Bergs, C. Bleilevens, G. Gayan-Ramirez, and C.S. Bruells, Sevoflurane exposure prevents diaphragmatic oxidative stress during mechanical ventilation but reduces force and affects protein metabolism even during spontaneous breathing in a rat model. Anesth Analg, 2015. 121(1): p. 73-80.

18. Pasqualin, R.C., C.T. Mostarda, L.E. Souza, M.F. Vane, R. Sirvente, D.A. Otsuki, M.L. Torres, M.C. Irigoyen, and J.O. Auler, Jr., Sevoflurane preconditioning during myocardial ischemia-reperfusion reduces infarct size and preserves autonomic control of circulation in rats. Acta Cir Bras, 2016. 31(5): p. 338-45.

19. Lu, Y., M. Bu, and H. Yun, Sevoflurane prevents hypoxia/reoxygenation-induced cardiomyocyte apoptosis by inhibiting PI3KC3-mediated autophagy. Hum Cell, 2019. 32(2): p. 150-159.

20. Zhong, H., H. Chen, and C. Gu, Sevoflurane Post-treatment Upregulated miR-203 Expression to Attenuate Cerebral Ischemia-Reperfusion-Induced Neuroinflammation by Targeting MyD88. Inflammation, 2020. 43(2): p. 651-663.

21. Fan, L., Y. Wu, J. Wang, J. He, and X. Han, Sevoflurane inhibits the migration and invasion of colorectal cancer cells through regulating ERK/MMP-9 pathway by up-regulating miR-203. Eur J Pharmacol, 2019. 850: p. 43-52.

22. Yuan, J. and Y. Zhang, Sevoflurane reduces inflammatory factor expression, increases viability and inhibits apoptosis of lung cells in acute lung injury by microRNA-34a-3p upregulation and STAT1 downregulation. Chem Biol Interact, 2020. 322: p. 109027. 
23. Wang, J., Y. Zhang, Y.M. Liu, L.L. Guo, P. Wu, Y. Dong, and G.J. Wu, Huoxue Anxin Recipe () promotes myocardium angiogenesis of acute myocardial infarction rats by up-regulating miR-210 and vascular endothelial growth factor. Chin J Integr Med, 2016. 22(9): p. 685-90.

24. Wang, N., C. Chen, D. Yang, Q. Liao, H. Luo, X. Wang, F. Zhou, X. Yang, J. Yang, C. Zeng, et al., Mesenchymal stem cells-derived extracellular vesicles, via miR-210, improve infarcted cardiac function by promotion of angiogenesis. Biochim Biophys Acta Mol Basis Dis, 2017. 1863(8): p. 2085-2092.

25. Bian, W.S., P.X. Shi, X.F. Mi, Y.Y. Sun, D.D. Yang, B.F. Gao, S.X. Wu, and G.C. Fan, MiR-210 protects cardiomyocytes from OGD/R injury by inhibiting E2F3. Eur Rev Med Pharmacol Sci, 2018. 22(3): p. 743749 .

26. Zhu, J., K. Lu, N. Zhang, Y. Zhao, Q. Ma, J. Shen, Y. Lin, P. Xiang, Y. Tang, X. Hu, et al., Myocardial reparative functions of exosomes from mesenchymal stem cells are enhanced by hypoxia treatment of the cells via transferring microRNA-210 in an nSMase2-dependent way. Artif Cells Nanomed Biotechnol, 2018. 46(8): p. 1659-1670.

27. Arif, M., R. Pandey, P. Alam, S. Jiang, S. Sadayappan, A. Paul, and R.P.H. Ahmed, MicroRNA-210mediated proliferation, survival, and angiogenesis promote cardiac repair post myocardial infarction in rodents. J Mol Med (Berl), 2017. 95(12): p. 1369-1385.

28. Liu, H., J. Wang, Y. Chen, Y. Chen, X. Ma, J.C. Bihl, and Y. Yang, NPC-EXs Alleviate Endothelial Oxidative Stress and Dysfunction through the miR-210 Downstream Nox2 and VEGFR2 Pathways. Oxid Med Cell Longev, 2017. 2017: p. 9397631.

29. Zhang, X., M. Li, G. Sun, Y. Bai, D. Lv, and C. Liu, MiR-563 restrains cell proliferation via targeting LIN28B in human lung cancer. Thorac Cancer, 2020. 11(1): p. 55-61.

\section{Figures}



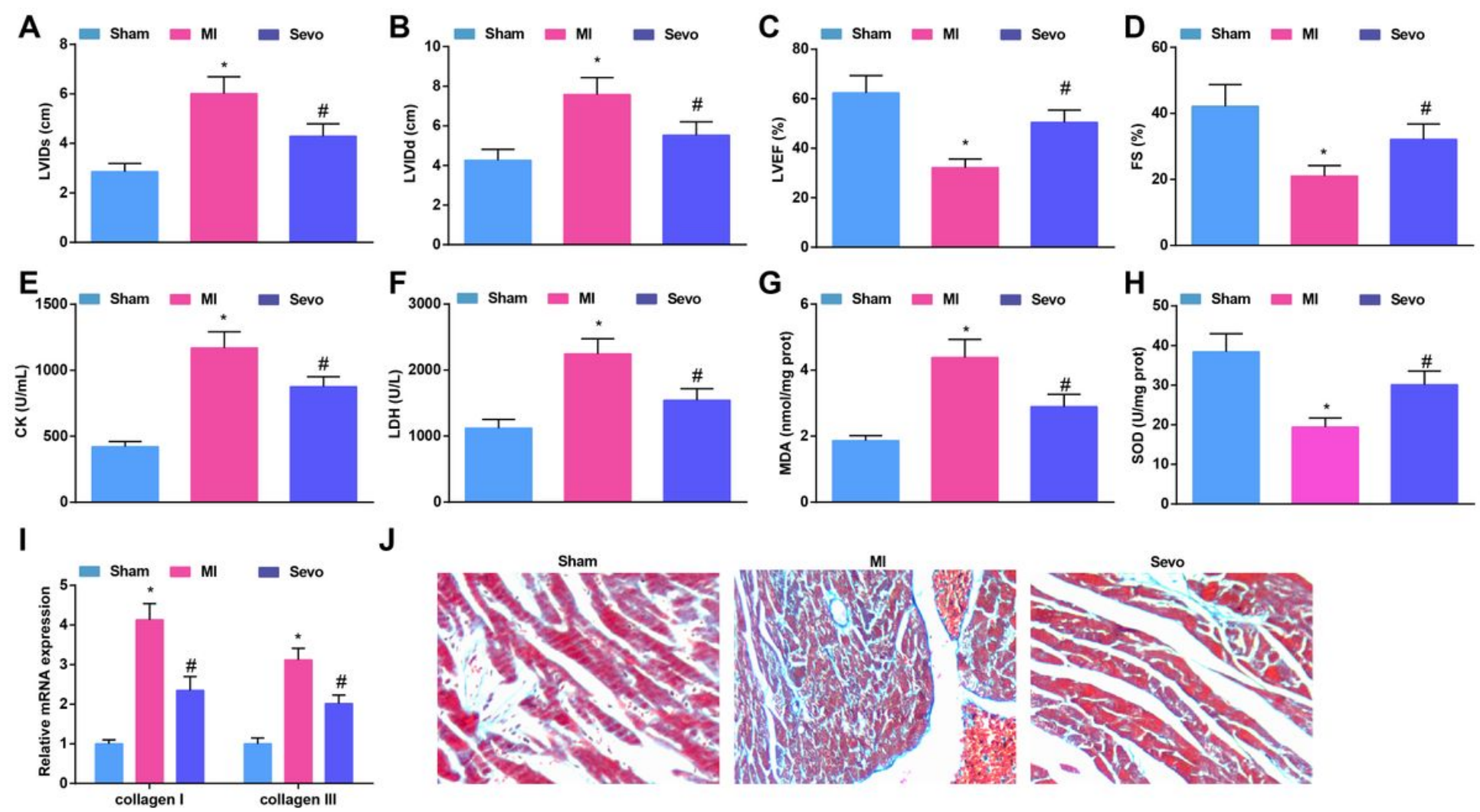

J
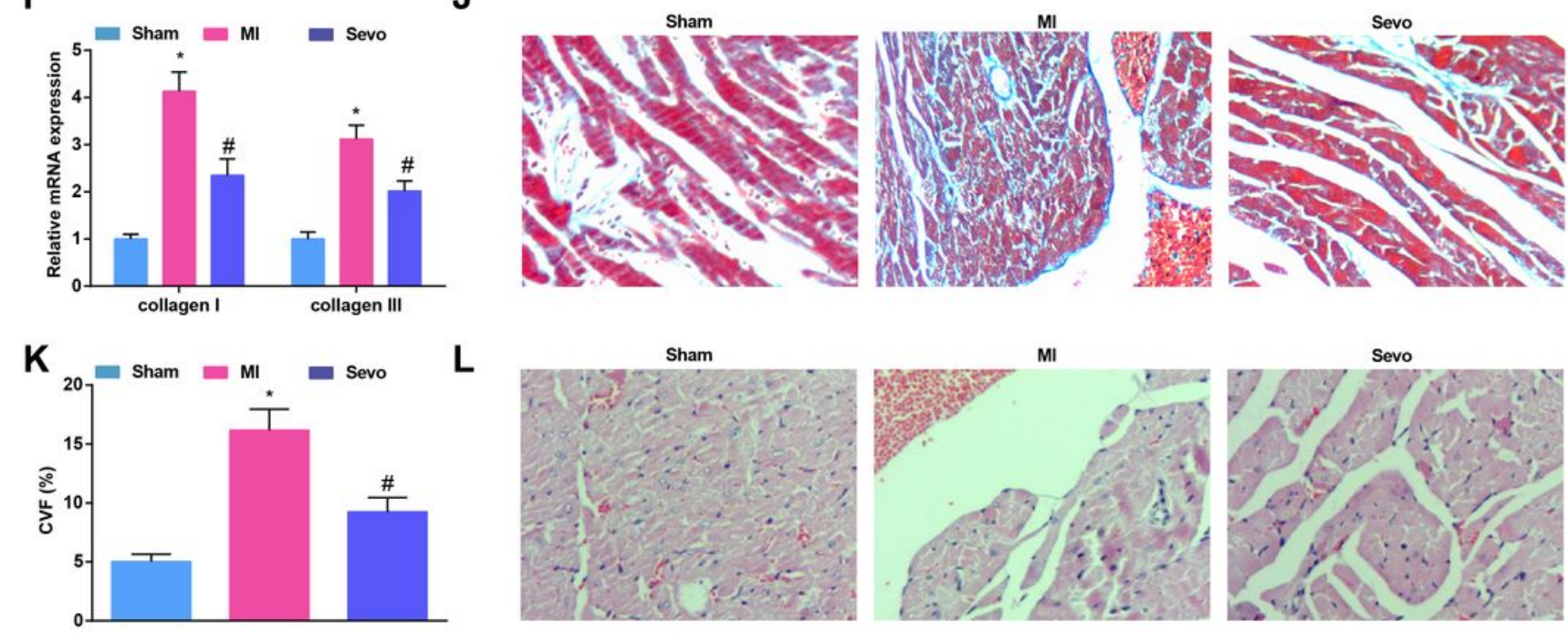

\section{M}
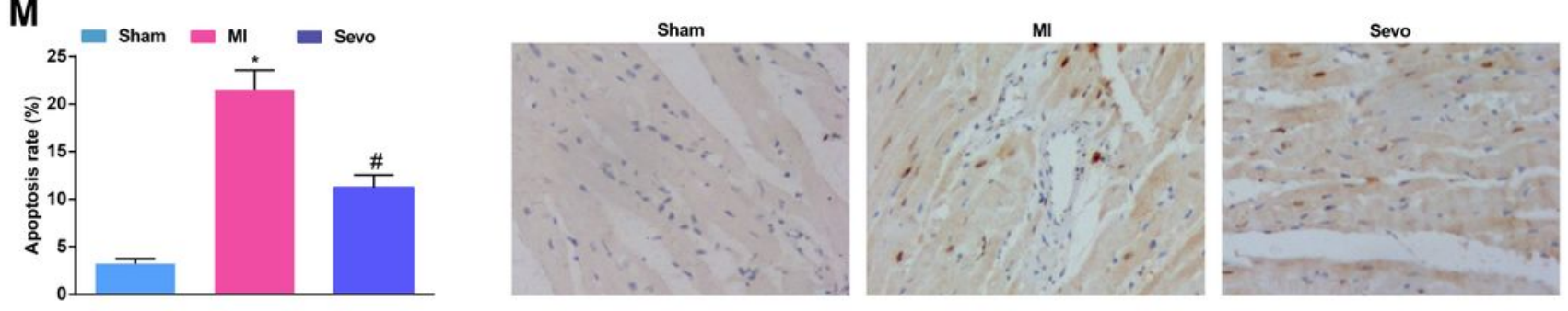

\section{Figure 1}

Sevo improves cardiac function of MI rats. A, LVIDs of rats in each group $(n=10) ; B, L V I D d$ of rats in each group $(n=10) ; C$, LVEF of rats in each group $(n=10) ; D$, FS of rats in each group $(n=10)$; E, serum CK activity of rats in each group; F, serum LDH activity of rats in each group; $G$, myocardial MDA activity of rats in each group; $\mathrm{H}$, myocardial SOD activity of rats in each group; I, mRNA expression of collagen I and collagen III in rat myocardial tissues was detected using RT-qPCR; J, representative images of Masson staining $(50 \mu \mathrm{m}) ; \mathrm{K}, \mathrm{CVF}$ of rat in each group; L, representative images of HE staining $(25 \mu \mathrm{m}) ; \mathrm{M}$, cardiomyocyte apoptosis in rat myocardial tissues was observed through TUNEL staining $(200 \mu \mathrm{m})$; *, P< 0.05 vs the sham group, \#, $P<0.05$ vs the Ml group; $n=5$, the measurement data conforming to the normal distribution were expressed as mean \pm standard deviation, one-way ANOVA was used for 
comparisons among multiple groups and Tukey's post hoc test was used for pairwise comparisons after one-way ANOVA.


\section{Figure 2}

MiR-210-3p downregulation eliminates sevo-induced effect on improving $\mathrm{Ml}$ in a rat model. A\&B, miR-210$3 p$ expression in rat myocardial tissues detected using RT-qPCR; C, LVIDs of rats in each group ( $n=10)$; D, LVIDd of rats in each group $(n=10)$; $E$, LVEF of rats in each group $(n=10)$; F, FS of rats in each group ( $n=$ 10); G, serum CK activity of rats in each group; $H$, serum LDH activity of rats in each group; I, myocardial MDA activity of rats in each group; J, myocardial SOD activity of rats in each group; K, mRNA expression of collagen I and collagen III in rat myocardial tissues was detected using RT-qPCR; L, representative images of Masson staining $(50 \mu \mathrm{m})$; M, CVF of rat in each group; $N$, representative images of HE staining $(25 \mu \mathrm{m})$; 0, cardiomyocyte apoptosis in rat myocardial tissues was observed through TUNEL staining $(200 \mu \mathrm{m}) ;{ }^{\wedge}, \mathrm{P}<0.05$ vs the sham group; \#, $\mathrm{P}<0.05$ vs the $\mathrm{Ml}$ group, ${ }^{*}, \mathrm{P}<0.05$ vs the sevo + antagomir $\mathrm{NC}$ group; $\mathrm{n}=5$, the measurement data conforming to the normal distribution were expressed as mean \pm standard deviation and the unpaired t-test was performed for comparisons between two groups, one-way 
ANOVA was used for comparisons among multiple groups and Tukey's post hoc test was used for pairwise comparisons after one-way ANOVA.
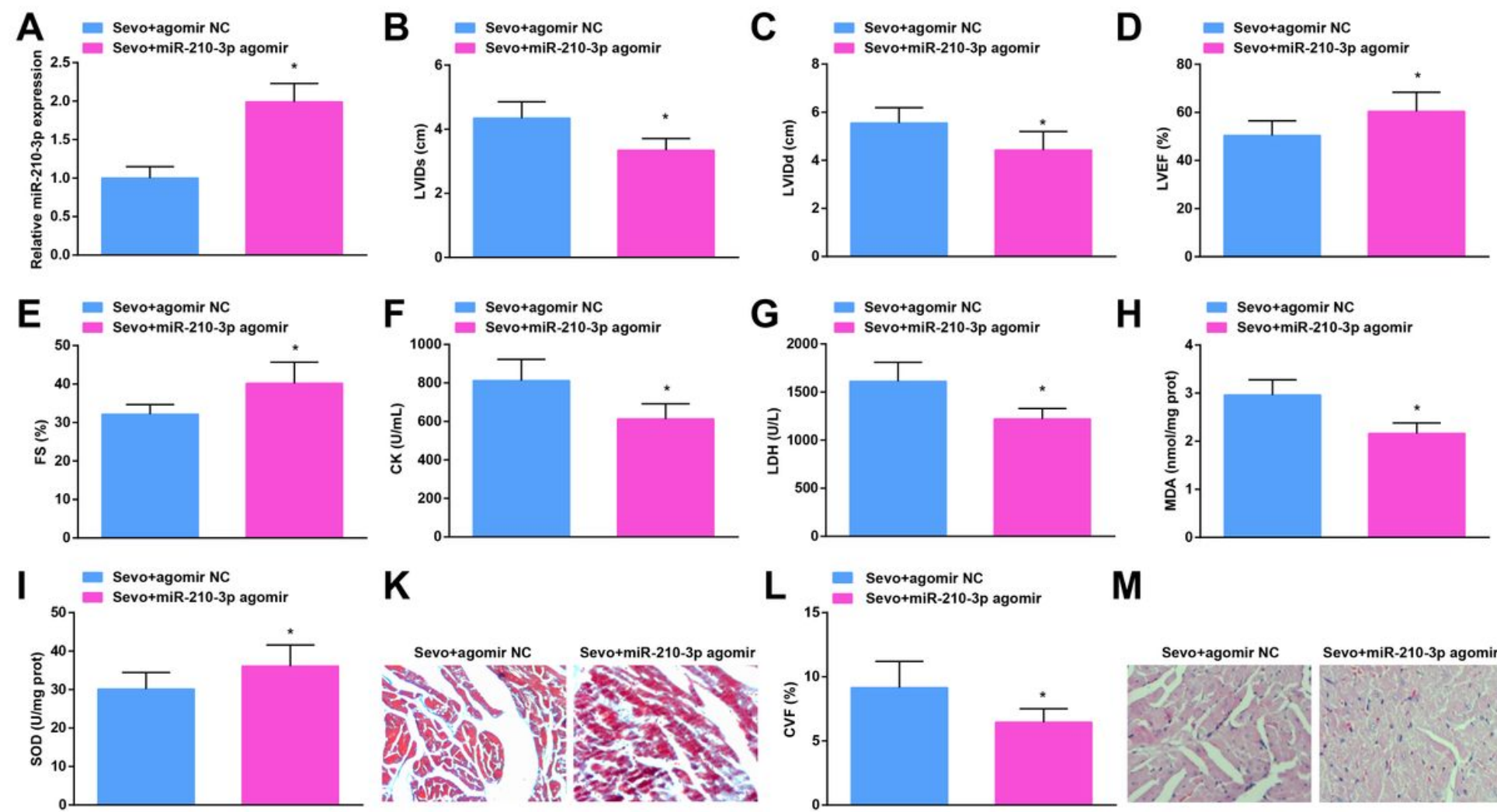

M
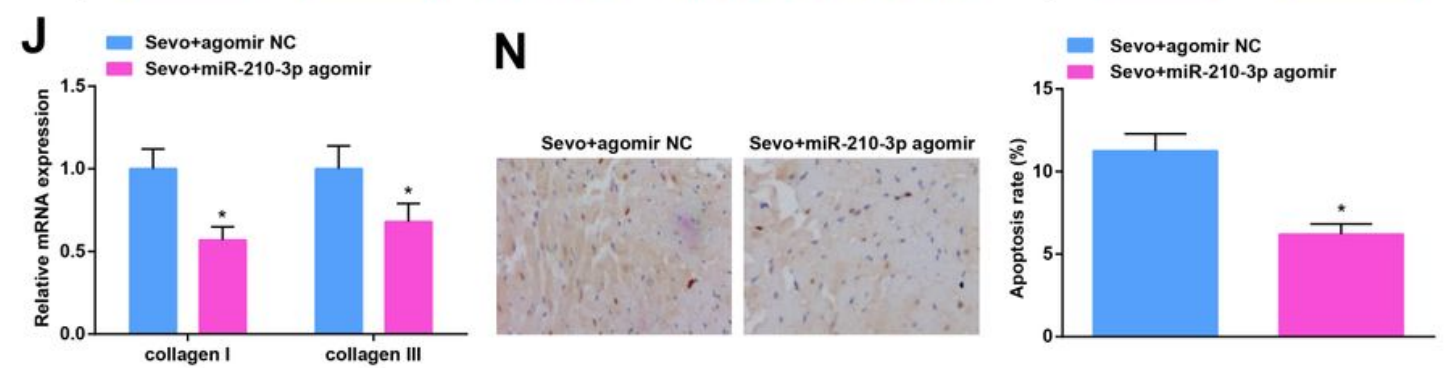

\section{Figure 3}

MiR-210-3p upregulation strengthens sevo-induced effect on improving $\mathrm{MI}$ in a rat model. A, miR-210-3p expression in rat myocardial tissues detected using RT-qPCR; B, LVIDs of rats in each group $(n=10)$; C, LVIDd of rats in each group $(n=10)$; D, LVEF of rats in each group $(n=10)$; E, FS of rats in each group ( $=10) ; \mathrm{F}$, serum CK activity of rats in each group; G, serum LDH activity of rats in each group; $\mathrm{H}$, myocardial MDA activity of rats in each group; I, myocardial SOD activity of rats in each group; J, mRNA expression of collagen I and collagen III in rat myocardial tissues was detected using RT-qPCR; K, representative images of Masson staining $(50 \mu \mathrm{m}) ; \mathrm{L}, \mathrm{CVF}$ of rat in each group; $\mathrm{M}$, representative images of HE staining $(25 \mu \mathrm{m})$; $\mathrm{N}$, cardiomyocyte apoptosis in rat myocardial tissues was observed through TUNEL staining $(200 \mu \mathrm{m}) ;{ }^{*}, \mathrm{P}<0.05$ vs the sevo + agomir NC group; $\mathrm{n}=5$, the measurement data conforming to the normal distribution were expressed as mean \pm standard deviation and the unpaired $t$ test was performed for comparisons between two groups. 


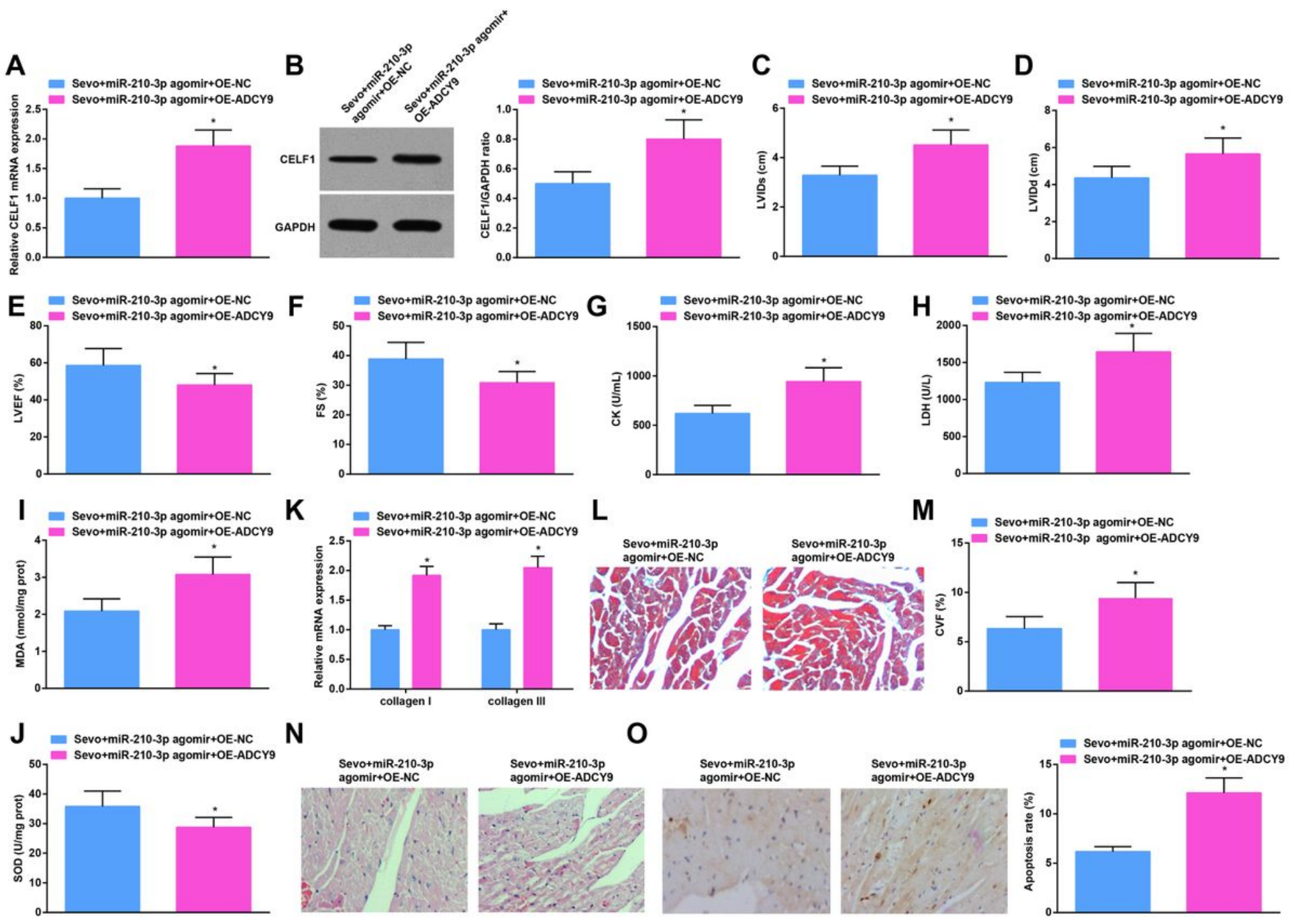

Figure 4

ADCY9 upregulation reverses the therapeutic effects of sevo and elevated miR-210-3p on MI rats. A, ADCY9 mRNA expression in rat myocardial tissues was detected using RT-qPCR; B, ADCY9 protein expression in rat myocardial tissues was detected using Western blot analysis; $C$, LVIDs of rats in each group $(n=10)$; D, LVIDd of rats in each group $(n=10)$; E, LVEF of rats in each group $(n=10) ; F$, FS of rats in each group $(n=10) ; G$, serum CK activity of rats in each group; $H$, serum LDH activity of rats in each group; I, myocardial MDA activity of rats in each group; J, myocardial SOD activity of rats in each group; $\mathrm{K}$, mRNA expression of collagen I and collagen III in rat myocardial tissues was detected using RT-qPCR; $L$, representative images of Masson staining $(50 \mu \mathrm{m}) ; \mathrm{M}, \mathrm{CVF}$ of rat in each group; $N$, representative images of HE staining $(25 \mu \mathrm{m})$; 0, cardiomyocyte apoptosis in rat myocardial tissues was observed through TUNEL staining $(200 \mu \mathrm{m}) ;{ }^{*}, \mathrm{P}<0.05$ vs the sevo + miR-210-3p agomir + oe-NC group; $\mathrm{n}=5$, the measurement data conforming to the normal distribution were expressed as mean \pm standard deviation and the unpaired t-test was performed for comparisons between two groups. 
A

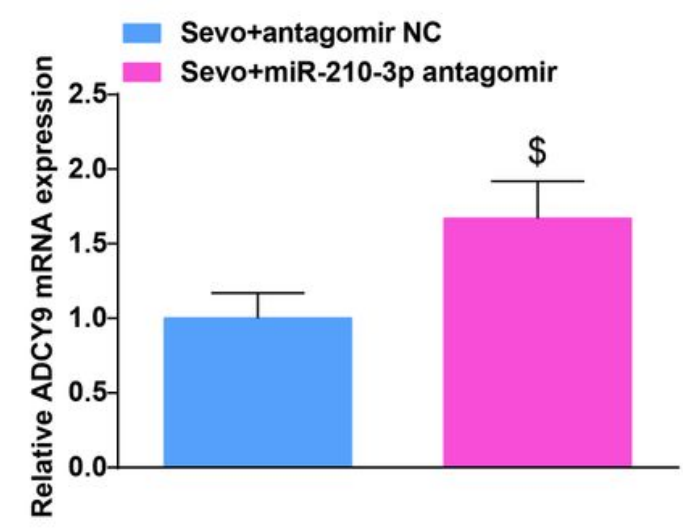

C

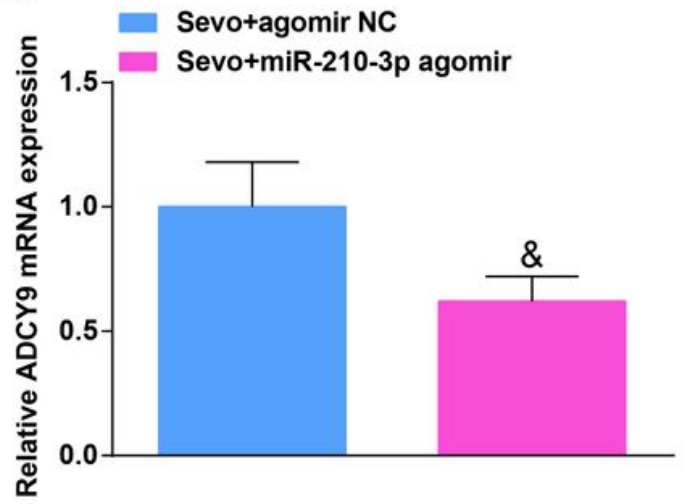

E
B

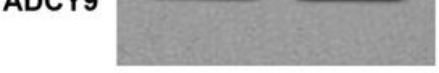

GAPDH

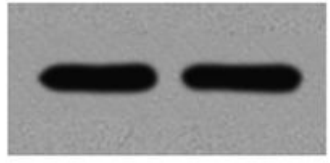

D

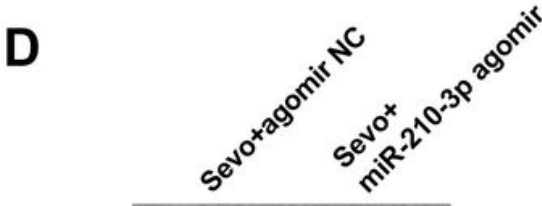

GAPDH

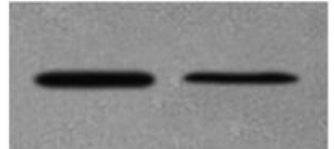

ADCY9

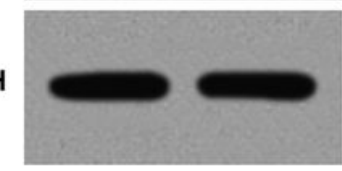

Sevo+antagomir NC

Sevo+miR-210-3p antagomir
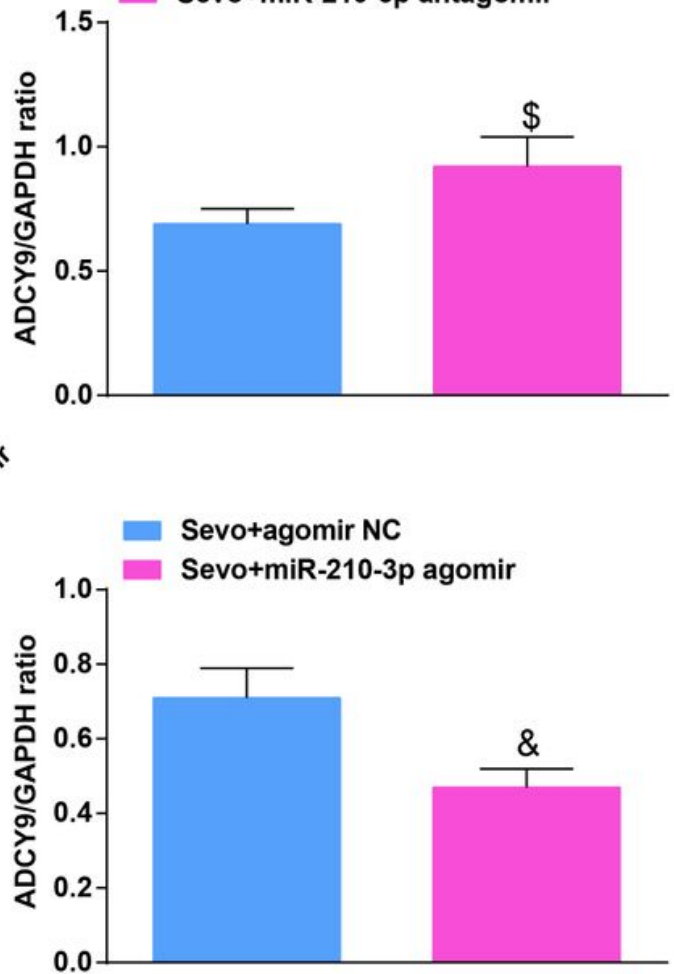

F

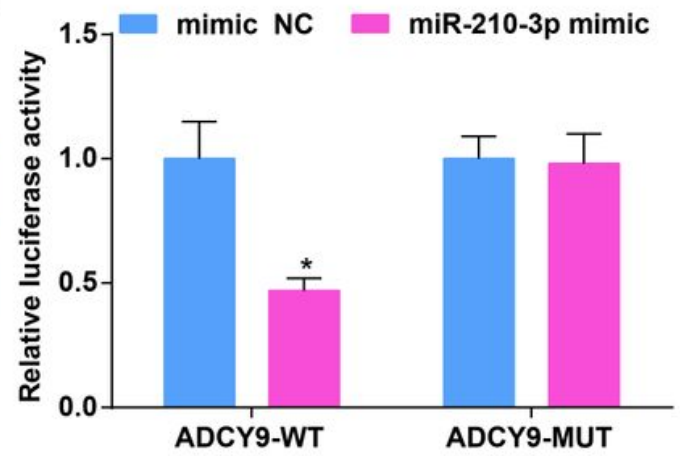

\section{Figure 5}

ADCY9 is a direct target gene of miR-210-3p. A, ADCY9 mRNA expression in the sevo + antagomir NC group and the sevo + miR-210-3p antagomir was detected using RT-qPCR; B, ADCY9 protein expression in the sevo + antagomir NC group and the sevo + miR-210-3p antagomir was detected using Western blot analysis; C, ADCY9 mRNA expression in the sevo + agomir NC group and the sevo + miR-210-3p agomir was detected using RT-qPCR; D, ADCY9 protein expression in the sevo + agomir NC group and the sevo + miR-210-3p agomir was detected using Western blot analysis; $E$, an online software was used to predict the binding sites of miR-210-3p and ADCY9; F, target relationship between miR-210-3p and ADCY9 was confirmed by dual luciferase report gene assay; $\$, P<0.05$ vs the sevo + antagomir NC group, \& $P<0.05$ vs the sevo + agomir NC group, ${ }^{*}, \mathrm{P}<0.05$ vs the mimic NC group; $N=3$; in Fig. A-D, data were analyzed 
using one-way ANOVA; in Fig. E\&F, data were analyzed using two-way ANOVA; Tukey's post hoc test was used for pairwise comparisons after ANOVA. 